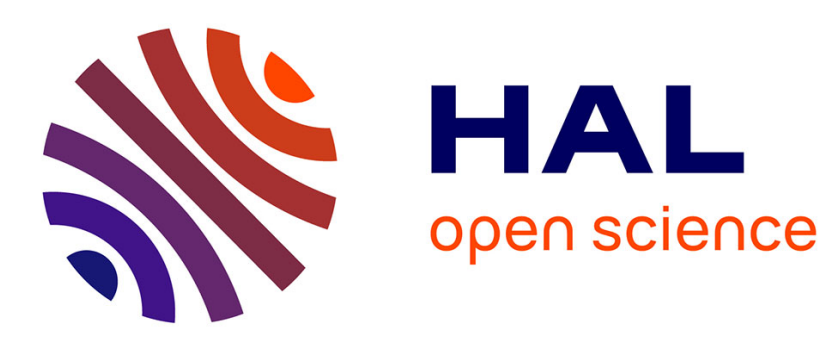

\title{
Analysis of Multi-elemental Thin Films via Calibration-Free Laser-Induced Breakdown Spectroscopy
}

Jörg Hermann, Emanuel Axente, Frédéric Pelascini, Valentin Craciun

\section{To cite this version:}

Jörg Hermann, Emanuel Axente, Frédéric Pelascini, Valentin Craciun. Analysis of Multi-elemental Thin Films via Calibration-Free Laser-Induced Breakdown Spectroscopy. Analytical Chemistry, 2019, 91 (3), pp.2544-2550. 10.1021/acs.analchem.8b05780 . hal-02114118

\author{
HAL Id: hal-02114118 \\ https://hal.science/hal-02114118
}

Submitted on 23 May 2019

HAL is a multi-disciplinary open access archive for the deposit and dissemination of scientific research documents, whether they are published or not. The documents may come from teaching and research institutions in France or abroad, or from public or private research centers.
L'archive ouverte pluridisciplinaire HAL, est destinée au dépôt et à la diffusion de documents scientifiques de niveau recherche, publiés ou non, émanant des établissements d'enseignement et de recherche français ou étrangers, des laboratoires publics ou privés. 


\title{
Analysis of multielemental thin films via calibration-free laser-induced breakdown spectroscopy
}

\author{
Jörg Hermann, ${ }^{*, \dagger}$ Emanuel Axente, ${ }^{\ddagger}$ Frédéric Pelascini, 9 and Valentin Craciun ${ }^{\ddagger}$ \\ Aix-Marseille University, CNRS, LP3, 13009 Marseille, France, National Institute for \\ Lasers, Plasma and Radiation Physics, 77125 Măgurele, Romania, and Cetim Grand Est, \\ 67305 Schiltigheim, France \\ E-mail: hermann@lp3.univ-mrs.fr
}

\section{Abstract}

Elemental analyses of thin films with complex composition are challenging as the standard analytical techniques based on measurement calibration are difficult to apply. We show that calibration-free laser-induced breakdown spectroscopy (LIBS) presents a powerful solution, enabling quantitative analyses of multielemental thin films with analytical performances better than those obtained with other techniques. The demonstration is given for a nickelchromium-molybdenum alloy film of $150 \mathrm{~nm}$ thickness that was produced by pulsed laser deposition. The LIBS spectra were recorded in experimental conditions that enable simple and accurate modeling of plasma emission. Thus, a calibration-free approach based on the calculation of the spectral radiance of a uniform plasma in local thermodynamic equilibrium was applied to deduce the elemental composition. Supported by analyses via Rutherford backscattering spectrometry and energy-dispersive Xray spectroscopy, the LIBS measurements evidence nonstoichiometric mass transfer of the alloy during the thin film deposition process.

\footnotetext{
*To whom correspondence should be addressed

${ }^{\dagger}$ Aix-Marseille University, CNRS, LP3, 13009 Marseille, France

$\ddagger$ National Institute for Lasers, Plasma and Radiation Physics, 77125 Măgurele, Romania

${ }^{\top}$ Cetim Grand Est, 67305 Schiltigheim, France
}

This technique could be used even for thinner films provided that the film-composing elements are not present in the substrate.

\section{Introduction}

Thin films attracted strongly growing interest during the last decades in many fields of applications such as optics, optoelectronics, biomedicine, and aeronautics. Consequently, great efforts have been dedicated to the development of techniques for thin film production and pulsed laser deposition (PLD) emerged as a versatile laboratory-based method after the successful synthesis of high-temperature supraconducting yttrium barium copper oxide layers in the late 80 's. ${ }^{1}$

The increasing demand of high-quality coatings with well-defined optical, electronic or mechanical properties stimulated the development of techniques for thin film characterization. Beside the determination of cristallographical structure and optoelectronical properties, the measurement of the elemental composition is of great interest. Rutherford backscattering spectrometry (RBS) and X-ray photoelectron spectrometry (XPS) are currently applied to measure elemental fractions within the deposited layer. ${ }^{2-4}$ According to the lower depth resolution, energy-dispersive X-ray spectrometry (EDS) is suitable for analysis of layers with 
thickness $\geq 1 \mu \mathrm{m}$. The analytical techniques based on the calibration with standard samples such as inductively coupled plasma mass or atomic emission spectrometry (ICP-MS or ICPAES) are inapplicable. ${ }^{5}$ Indeed, ICP analyses are typically used for liquid sampling, while extensive sample preparation (dissolution) is required for solids. ${ }^{6}$ Although direct ICP analysis of solids is possible via laser ablation (LA) sampling, LA-ICP measurements are affected by difficulties related to sample size, shape, heterogeneity, and the lack of matrix-matched standards. $^{7}$

Beside the cost- and time-expensive use of RBS and XPS analyses, these techniques are limited to materials having a relatively simple elemental composition. ${ }^{8}$ Moreover, RBS and XPS suffer low sensitivity and elements cannot be accurately measured in many cases if their mass fraction within the thin film is below $1 \%$. The poor limit of detection is usually not a handicap in thin film analysis as the deposited layers mostly have a simple elemental composition: they include a few elements only with relative large abundances.

However, some materials such as wear- or corrosion-resistant coatings have more complex elemental composition. Among them, superalloys such as nickel-chromium-molybdenum alloys are of great interest. Their tribological properties critically depend on the elemental composition and accurate compositional analyses including major and minor elements are required for quality control.

As an emerging technique for fast multielemental analysis, laser-induced breakdown spectroscopy was proposed for thin film analysis. ${ }^{9}$ However, due to the ablation depth being typically larger than several hundreds of nanometers, accurate LIBS analyses based on calibration with standard samples were limited to films of thickness $\geq 1 \mu \mathrm{m} .{ }^{10}$ To overcome the difficulties due to calibration, thin films were analyzed via calibration-free LIBS methods. ${ }^{11-13}$ These techniques are based on modeling of the emission spectrum of the laser-produced plasma. ${ }^{12,14}$ The reported calibration-free analyses only concern thin films of simple composition, containing 3 elements at maximum. More- over, these analyses were mostly carried out with very simple approaches that ignore selfabsorption and thus suffer from low accuracy. ${ }^{15}$ In the present paper, we demonstrate that quantitative analysis of thin films with complex composition are possible via calibrationfree LIBS measurements based on accurate and robust modeling. The method takes benefit from experimental conditions that were recently found to generate a plume well described by the simple model of a uniform plasma in local thermodynamic equilibrium. ${ }^{16}$

\section{Experimental section}

\section{LIBS setup}

The experiments were carried out with Nd:YAG laser pulses of $6 \mathrm{~mJ}$ energy and $4 \mathrm{~ns}$ duration. The laser was operated in the ultraviolet spectral range $(266 \mathrm{~nm})$ to obtain efficient energy coupling towards the sample material. The beam was focused to a spot of $100 \mu \mathrm{m}$ diameter on the sample surface, leading to a laser fluence of about $100 \mathrm{~J} \mathrm{~cm}^{-2}$ that is high enough to ensure stoichiometic ablation. ${ }^{17}$ The samples were placed on a motorized sample holder in a vacuum chamber that was filled with argon at $5 \times 10^{4} \mathrm{~Pa}$ pressure.

Optical emission spectroscopic measurements were performed by imaging the plasma with two lenses of 150 and $35 \mathrm{~mm}$ focal lengths onto the entrance of an optical fiber of $600 \mu \mathrm{m}$ core diameter. The fiber was coupled to the entrance of an echelle spectrometer with a resolving power of $9 \times 10^{3}$. Photon detection was ensured using an intensified charge-coupled device matrix detector. The apparatus spectral width and the apparatus response were measured as functions of wavelength with appropriate calibrated lamps.

The spectra were recorded with a gate width $\Delta t_{g}$ short enough to make sure that the variations of electron density and plasma temperature during the recording were small compared to their absolute values. Typically, $\Delta t_{g} \leq t_{d} / 2$ was applied, where $t_{d}$ is the delay between the laser pulse and the detector gate. We denote 
Table 1: Mass fraction deduced from the LIBS analysis $C_{L I B S}$, atomic mass $m_{A}$, and first ionization potential $\Delta_{i o n}$ of elements composing the nickel-chromium-molybdenum alloy (Hart b.v., Inconel 625) used for thin film deposition. The $C_{L I B S}$-values correspond to the recording delay of highest measurement accuracy (see Fig. 7).

\begin{tabular}{cccc}
\hline Element & $C_{L I B S}(\%)$ & $m_{A}(\mathrm{amu})$ & $\Delta_{i o n}(\mathrm{eV})$ \\
\hline $\mathrm{Ni}$ & $63.1 \pm 3.6$ & 58.70 & 7.64 \\
$\mathrm{Cr}$ & $21.2 \pm 3.1$ & 52.00 & 6.77 \\
$\mathrm{Mo}$ & $9.4 \pm 1.4$ & 95.94 & 7.09 \\
$\mathrm{Nb}$ & $3.7 \pm 1.2$ & 92.91 & 6.76 \\
$\mathrm{Fe}$ & $1.7 \pm 0.3$ & 55.85 & 7.90 \\
$\mathrm{Mn}$ & $0.07 \pm 0.03$ & 54.94 & 7.43 \\
$\mathrm{Si}$ & $0.35 \pm 0.10$ & 28.09 & 8.15 \\
$\mathrm{Al}$ & $0.30 \pm 0.07$ & 26.98 & 5.99 \\
$\mathrm{Ti}$ & $0.24 \pm 0.05$ & 47.90 & 6.83 \\
$\mathrm{Co}$ & $0.10 \pm 0.03$ & 58.93 & 7.86 \\
$\mathrm{Ca}$ & $0.018 \pm 0.005$ & 40.08 & 6.11 \\
\hline
\end{tabular}

the time of observation $t=t_{d}+\Delta t_{g} / 2$. To enhance the signal-to-noise ratio, data acquisition was performed by averaging over 100 ablation events, applying a single pulse on each irradiation site. We note that the present experiments were carried out with a spectroscopic apparatus of low efficiency (echelle spectrometer) and that the acquisition number can be reduced to a few repetitions if a more efficient system is used. The ablation depths measured via optical microscopy were 0.55 and $1.0 \mu \mathrm{m}$ for the bulk sample and the deposited thin film, respectively (see SI 1).

The thin film was produced via pulsed laser deposition onto an electronic grade low resistivity Si (100) substrate of $99.999 \%$ purity. The film thickness of $150 \mathrm{~nm}$ was deduced from RBS analysis (see Fig. S1). A detailed description of the PLD arrangement can be found in a previous paper. ${ }^{18}$ The alloy target is irradiated in a vacuum chamber at $10^{-5} \mathrm{~Pa}$ pressure. Here, a moderate laser fluence of $2 \mathrm{~J} \mathrm{~cm}^{-2}$ is applied to obtain a smooth film. The nickel-chromiummolybdenum alloy (see Table 1) was chosen as sample material for its complex multielemental composition.

\section{Computational details}

Material ablation with ultraviolet laser pulses of nanosecond duration in a near-atmospheric argon atmosphere generates a plasma with ideal properties for spectroscopic measurements, combining two attributes that are usually not found together: the plasma is (i) spatially uniform and (ii) well described by the model of local thermodynamic equilibrium (LTE). ${ }^{16}$ Consequently, the spectral radiance of the plasma can be calculated using a simple analytical solution of the radiation transfer equation. ${ }^{19}$

We have implemented the calculation of the spectral radiance in an iterative measurement procedure that allows us to deduce the elemental composition from the best agreement between measured and computed spectra. ${ }^{20}$ The procedure consists of two main measurement loops. The principal loop includes the successive measurements of electron density $n_{e}$, plasma temperature $T$ and the fractions $C_{i}$ of the $N$ elements. For each measurement, the corresponding parameter is varied in order to find the best agreement between the computed and measured spectra in the wavelength ranges of the relevant atomic or ionic transitions. The calculations include the precise description of the spectral line profile, considering the dominating line broadening effects, namely Doppler and Stark broadening. For spectral lines of unknown Stark broadening parameters, the Stark width and shift are measured in a separate experiment. ${ }^{21,22}$ The procedure also includes an algorithm for the selection of the most appropriate lines according to their optical thickness, signal-to-noise ratio, interference with other transitions, and the accuracy of available spectroscopic data. The data were taken in priority from the NIST database ${ }^{23}$ and completed by data from the Kurucz database. ${ }^{24}$

The calibration-free measurement algorithm was successfully validated for glasses and alloys. ${ }^{20,25}$ It was shown that the model validity and thus the composition measurement accuracy critically depend on the time of observation. For elements such as oxygen, characterized by an atomic structure with large energy gaps between the electronic excitation levels, 


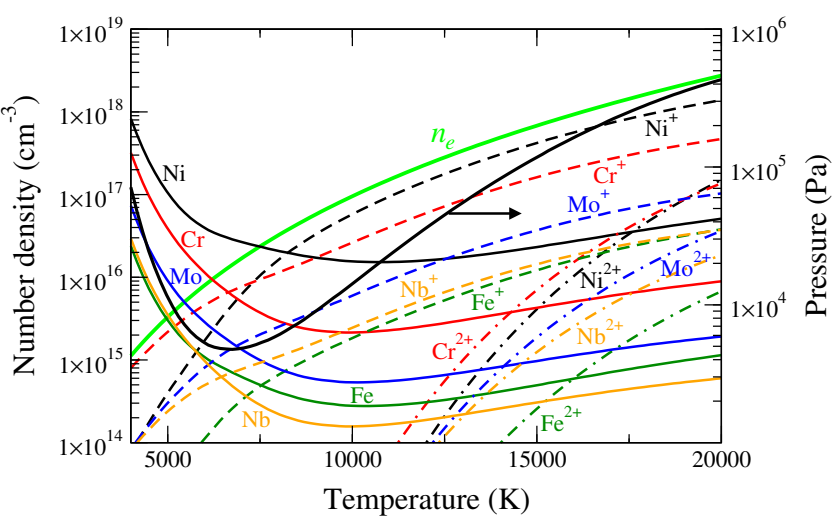

Figure 1: Number densities of species vs temperature computed for a plasma in LTE with the elemental composition of the Inconel 625 alloy (see Table 1). The calculations were performed for the $n_{e}=f(T)$-dependence deduced from the measurements (see Fig. 4), assuming a negligible fraction of argon in the vapor plasma. The calculated kinetic pressure is presented with respect to the secondary y-axis.

the equilibrium condition is only fulfilled for $t<1 \mu \mathrm{s}$, when $n_{e}>1 \times 10^{17} \mathrm{~cm}^{-3} \cdot{ }^{20,25}$ Contrarily, metal atoms typically have many excitation levels separated by small energy gaps. The lifetime of local thermodynamic equilibrium is therefore significantly longer in case of metallic plasmas. ${ }^{25,26}$

The number densities of plasma species computed for LTE are displayed in Fig. 1 as functions of temperature for the elemental composition of the considered nickel-chromiummolybdenum alloy. For a clear presentation, only the most abundant elements are displayed. According to the moderate differences in ionization energy (see Table 1 ), the $T$-dependence of atomic and ionic number densities is similar for all elements. For $T<7000 \mathrm{~K}$, neutral atoms dominate the plasma composition. Their number densities are shown to reach a minimum for $T \simeq 10000 \mathrm{~K}$. The increase of the atomic number densities with temperature for $T>10000 \mathrm{~K}$ is attributed to the high density of the laser-produced plasma during the early expansion stage. ${ }^{27}$ The high density favors the recombination processes, and the temperature required to obtain a given degree of ionization increases with density. For the same reason, doubly charged ions are generally not observed in LIBS plasmas. The pressure of the plasma is shown to reach a minimum for $T \simeq 7000 \mathrm{~K}$. The minimum below the ambient gas pressure is expected after the moment when the plasma reaches the maximum volume. The recompression afterwards is illustrated by the increase of pressure in the low-temperature range, similar to the implosion that succeeds a classical explosion. $^{27}$

\section{Results and discussion}

The calibration-free LIBS measurement procedure was applied to spectra recorded with different gate delays in order to find the observation time for highest analytical performance. The electron density was deduced from Stark broadening of spectral lines having known Stark broadening parameters, assuming a linear dependence between Stark width and electron density. ${ }^{28}$ As an example, the spectral profiles of the Ca II $393.36 \mathrm{~nm}$ transition (see Table S1) measured for different delays are displayed in Fig. 2 together with the computed line profiles. According to the low fraction of calcium (see Table 1) the self-absorption of the resonance line is small and $n_{e}$ can be measured with fair accuracy for $t \leq 1 \mu \mathrm{s}$, when the Stark width is large enough.

Taking into account all sources of measurement errors, the accuracy of $n_{e}$-measurements

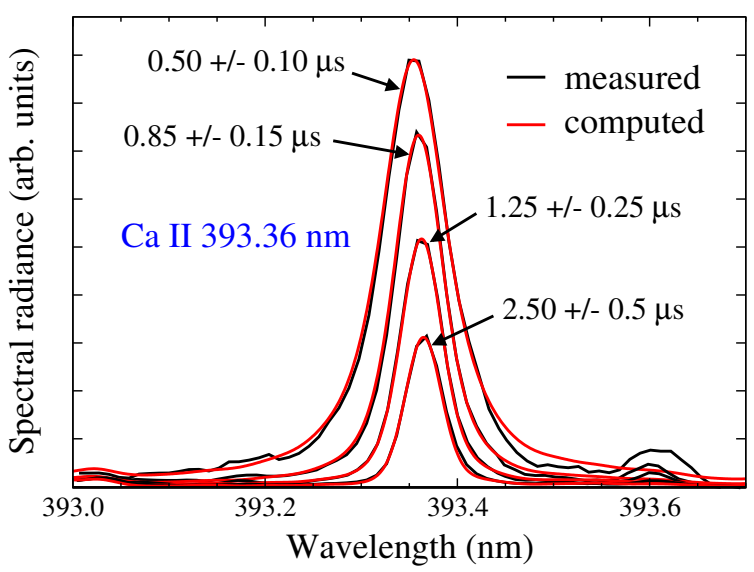

Figure 2: Measured and computed profiles of Ca II $393.36 \mathrm{~nm}$. The recordings were performed during ablation of the bulk alloy sample for different times of observation. 


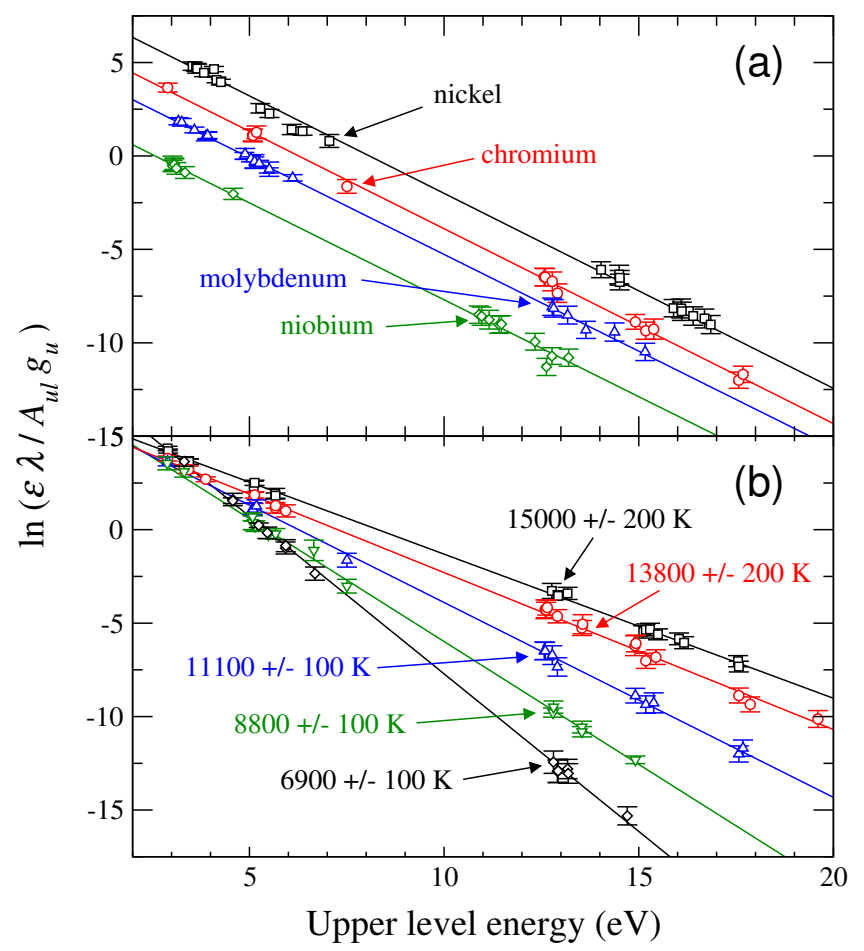

Figure 3: Saha-Boltzmann plots (a) for most abundant elements at $t=0.85 \mu \mathrm{s}$, and (b) for chromium at $0.35 \mu \mathrm{s}$ (squares), $0.5 \mu \mathrm{s}$ (circles), $0.85 \mu \mathrm{s}$ (triangles up), $2.5 \mu \mathrm{s}$ (triangles down), and $6.0 \mu \mathrm{s}$ (diamonds). In the logarithmic function, $\varepsilon, \lambda, A_{u l}$ and $g_{u}$ are the emission coefficient, the wavelength, the transition probability and the upper level statistical weight of the lines, respectively.

is estimated to about $20 \%$ in the time interval from 0.5 to $2 \mu \mathrm{s}$. At later times, the measurement uncertainty increases as the Stark widths of most lines become small compared to the apparatus spectral width while an decrease of accuracy due to the simplified theoretical description (temperature dependence is ignored) is expected for shorter times.

The alloy-forming most abundant elements are characterized by rich emission spectra, but low accuracy of the available spectroscopic data. Thus, to reduce measurement errors due to inaccurate data, a large number of lines was included in the analysis. This is illustrated by the Saha-Boltzmann plot displayed in Fig. 3 (a) where the parallel slopes indicate the high accuracy of the deduced temperature values. The Saha-Boltzmann plots obtained for chromium at different times (b) show that accurate $T$ -

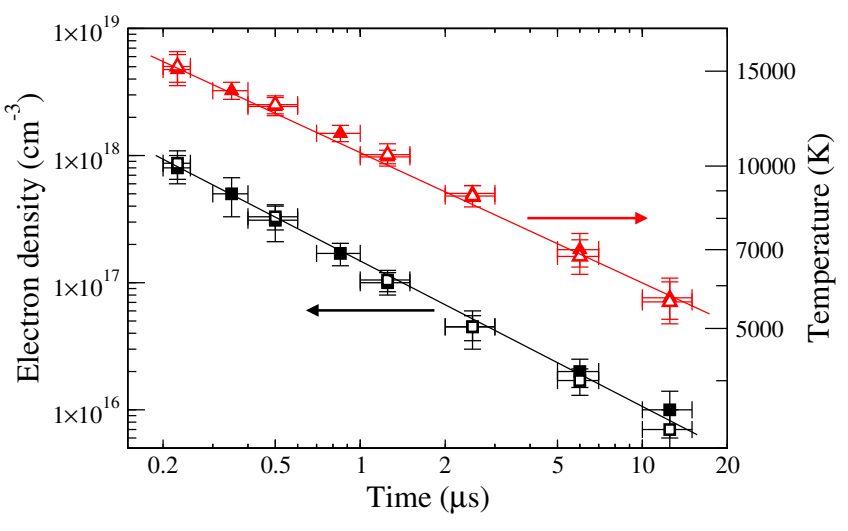

Figure 4: Electron density and temperature vs time measured for the bulk sample (filled symbols) and the thin film (empty symbols). The vertical error bars represent the measurement uncertainties whereas the horizontal error bars indicate the detector gate width.

measurements were achieved over the entire considered time interval.

The electron density and plasma temperature measured for both the bulk sample and the deposited thin film are displayed in Fig. 4 as functions of time. It is shown that the properties of the plasma produced in both cases are equal within the measurement uncertainties. This behaviour is expected for laser ablation with fluences strongly above the ablation threshold where the properties of the solid material play a minor role and the breakdown process is mainly governed by the attributes of the ablated vapor atoms. The time evolution of $n_{e}$ and $T$ is approximated by functions $f(t)=A t^{B}$ (continuous lines) with the parameters $A$ and $B$ deduced from the best fit. We obtain $n_{e}(t)=$ $2.19 \times 10^{20} t^{-1.07}$ and $T(t)=4.93 \times 10^{4} t^{-0.22}$, where $t, n_{e}$ and $T$ are in units of ns, $\mathrm{cm}^{-3}$ and $\mathrm{K}$, respectively. The combination of both expressions gives the temperature dependence of electron density $n_{e}(T)=0.0038 T^{4.85}$ that was used as boundary condition for the calculations of the LTE plasma composition displayed in Fig. 1.

The capability of compositional measurements is illustrated by the spectra displayed in Fig. 5. The two selected spectral ranges exhibit transitions of the most abundant elements of the nickel-chromium-molybdenum alloy. Compared to the line intensity distribution 


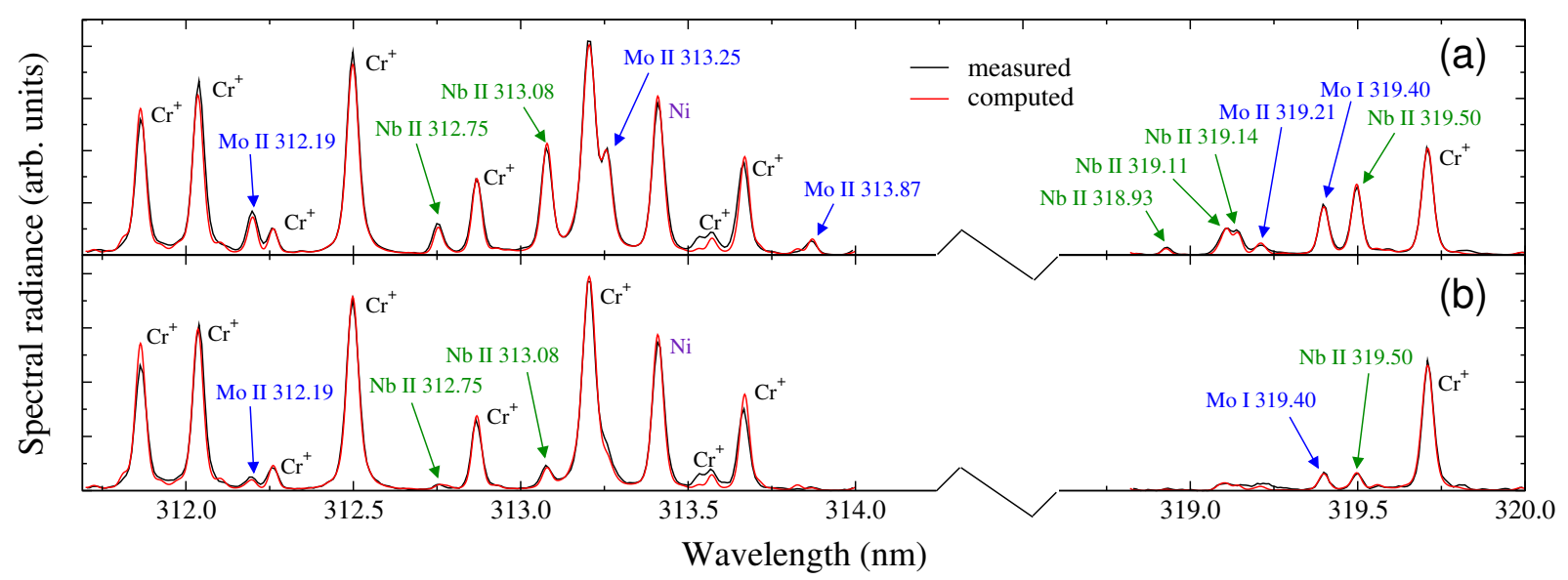

Figure 5: Measured and computed spectral radiance of transitions from chromium, molybdenum, niobium and nickel. The recordings were performed during ablation of the bulk alloy sample (a) and the deposited thin film (b) for $t=1.25 \pm 0.25 \mu \mathrm{s}$. The spectral lines used for the composition measurements are listed in Table S1.

observed for the bulk sample (a), the relative intensities of $\mathrm{Mo}$ and $\mathrm{Nb}$ transitions with respect to $\mathrm{Ni}$ or $\mathrm{Cr}$ lines are much lower in the spectrum recorded for the thin film (b). We deduce a significant change of composition, indicating a nonstoichiometric mass transfer during the pulsed laser deposition process. The deficiency of molybdenum and niobium by a factor of four was confirmed by analysis of spectra recorded with different gate delays (see Tables S2 and S3). The good agreement between measured and computed spectra indicates the high accuracy of calibration-free LIBS analysis.

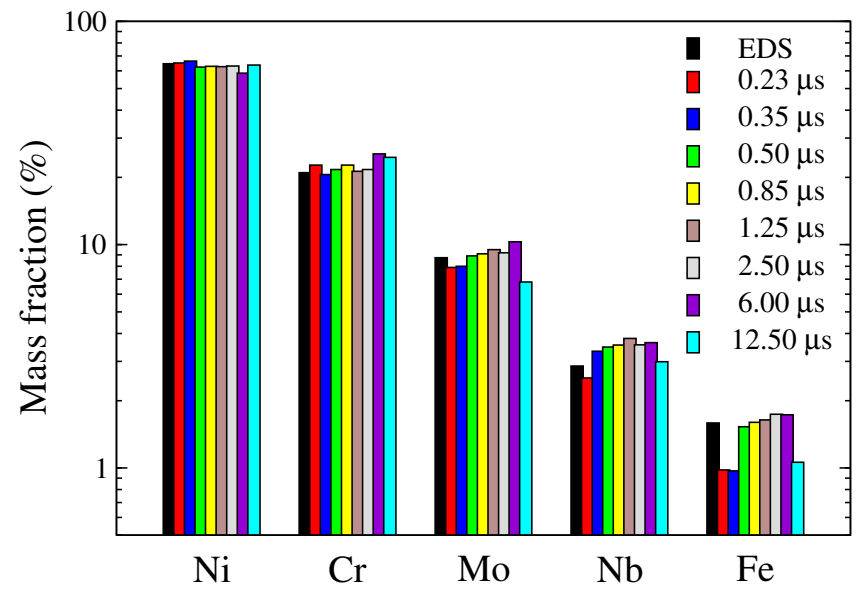

Figure 6: Mass fractions of most abundant elements deduced for the Inconel 625 sample from spectra recorded with different gate delays (see Table S2). The reference values (black columns) were measured via EDS.
The mass fractions of the most abundant elements measured for the bulk alloy are presented in Fig. 6 for various measurement times. The almost constant element fractions indicate the validity of the LTE model over the entire considered time interval, in accordance with previous analysis of aluminum. ${ }^{25} \mathrm{~A}$ good agreement with the EDS reference values is observed for the calibration-free LIBS analysis of spectra recorded from $t=0.5$ to $6 \mu \mathrm{s}$.

To find the gate delay for most accurate analysis, we estimated the fraction measurement

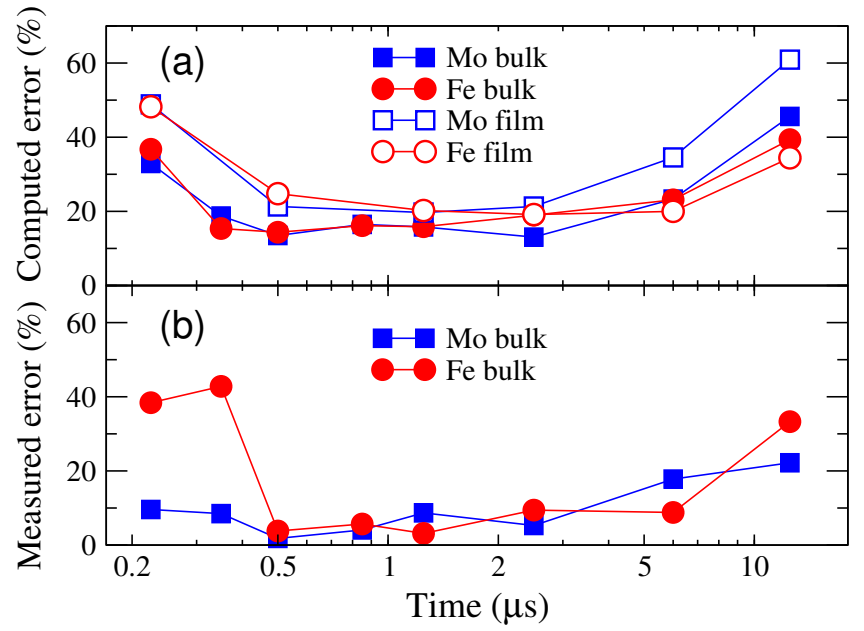

Figure 7: Relative errors of Mo and Fe fraction measurements vs measurement time (a) predicted by the calibration-free LIBS algorithm and (b) evaluated from the difference with the values measured via EDS. 
errors for all measurement times. The characteristic time evolution is displayed in Fig. 7 for molybdenum and iron. According to their abundances, the measurement errors of Mo and Fe are representative for elements with mass fractions of the order of $10 \%$ and $1 \%$, respectively.

The errors deduced from the spectra simulation are presented for both the bulk sample and the thin film (a). The error evaluation takes into account all relevant error sources, including (i) intensity measurement errors due to signalto-noise ratio and uncertainty of the apparatus response, (ii) accuracy of spectroscopic data and, (iii) self-absorption. For both elements, the most accurate measurements were achievable in the time interval from 1 to $2 \mu \mathrm{s}$. For smaller recording times, the measurement error increases mostly due to enhanced Stark broadening and the increase of the continuum emission intensity. This leads to stronger line interferences and lower signal-to-noise ratio. For $t>2 \mu \mathrm{s}$, the self-absorption of the most intense lines (having small upper level energies) increases. Thus, the measurements have to be performed with lines of reduced intensities for which the signal-to-noise ratio is low. In addition, the uncertainty of the electron density measurement increases as a consequence of line narrowing. The minimum error of about 10 to $15 \%$ obtained for the intermediary time interval is mainly due to the uncertainty of the available spectroscopic data.

The measurement errors evaluated from the difference between the mass fractions measured by LIBS and those obtained via EDS are displayed in Fig. 7 (b) for the bulk sample. Similar to the behavior of the computed errors (a), the time evolution of the measured errors (b) reach a minimum located in the time interval from 1 to $2 \mu \mathrm{s}$. However, the measured errors are systematically smaller, indicating that the accuracy of the used spectroscopic data is better than the given uncertainty. The measured error is not presented for the thin film (b) as both RBS and EDS analysis had too low accuracy. A further reduction of the measurement error down to $5 \%$ could be readily expected with the availability of more accurate spectroscopic data.

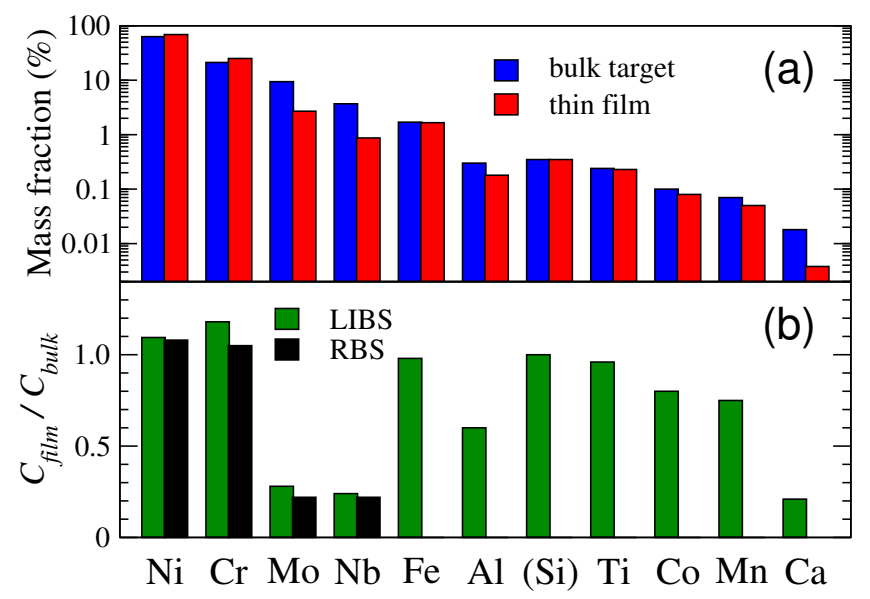

Figure 8: (a) Elemental compositions of the bulk alloy target and the thin film deduced from the spectra recorded for $t=1.25$ and $2.50 \mu \mathrm{s}$. (b) The deduced fraction ratio $C_{f i l m} / C_{b u l k}$ is compared the to ratio measured via RBS for the most abundant elements (see Table S4).

The elemental compositions measured for both the bulk alloy sample and the thin film are displayed in Fig. 8 (a). To illustrate the characteristic changes, the fraction ratios $C_{f i l m} / C_{b u l k}$ are presented in (b). The amount of silicon (in parentheses) within the thin film could not be measured due to the contribution of the Si substrate to the LIBS signal. The Si-fraction was thus set equal to the value measured for the bulk sample. We emphasize that this adjustment is done for the presentation of the final result only. The LIBS measurements procedure was operated with the silicon amount from both thin film and substrate.

A good agreement with the fraction ratios measured via RBS is observed for the most abundant elements for which RBS measurements were possible (see Fig. S1). Compared to their abundance in the bulk target, the fractions of molybdenum and niobium in the film are strongly reduced. Accordingly, the amount of the major elements (nickel and chromium) is slightly increased.

The deficiency of Mo and Nb in the thin film evidences a nonstoichiometric mass transfer during the pulsed laser deposition process. This phenomenon is often observed in PLD and explained by different mechanisms. Among them, the mass-dependent (see Table 1) angular dis- 
tributions of atoms and ions in the plume and non-stoichiometric ablation have been reported. ${ }^{29,30}$ Operated with low laser fluence to minimize droplet generation, the ablation mechanism in PLD is close to thermal evaporation for which the elemental compositions in the liquid and in the gas phase differ as a consequence of the element-dependent values of boiling temperature and vaporization enthalpy (in Table S6). ${ }^{17}$ It was recently shown that the composition of a thin film deposited via PLD from a copper-tungsten alloy target could be tuned from pure copper to $97 \%$ tungsten by changing laser fluence and angular position of the substrate. ${ }^{31}$ The mass-dependent sticking probability of atoms and ions on the thin film was also considered. ${ }^{32,33}$ In the present experiment, the contribution of nonstoichiometric vaporization is indicated by EDS analysis that show an enrichment of molybdenum and niobium on the alloy target in the zone irradiated by the laser during pulsed laser deposition (see Table S5, Figs. S2 and S3).

The calcium fraction within the thin film is also found to be significantly smaller than the value measured for the bulk sample. This change is attributed to the nonuniform distribution of $\mathrm{Ca}$ within the bulk sample, characterized by a maximum value at the surface and a rapid decrease as a function of depth (see Fig. S4).

\section{Conclusion}

The present results demonstrate that compositional analyses of multielemental thin films can be performed via calibration-free laser-induced breakdown spectroscopy with analytical performances better than those obtained with other techniques. The demonstration was given for a nickel-chromium-molybdenum alloy composed of five elements with mass fractions $>1 \%$ and several elements of lower abundance. To ensure simple and accurate modeling of the plasma emission spectrum, the LIBS experiments were carried out with ultraviolet nanosecond laser pulses in a near-atmospheric argon pressure. These conditions were previously shown to enable the formation of a uniform plasma in lo- cal thermodynamic equilibrium. Analyses performed for different delays between the laser pulse and the detector gate reveal that the most accurate measurements are obtained in the time interval from 1 to $2 \mu \mathrm{s}$. In this condition, the measurement accuracy is mostly limited by the uncertainty of the used spectroscopic data, and the reported errors of about $15 \%$ can be reduced down to $5 \%$ if accurate data are available.

Analyzing both the bulk alloy sample and the $150 \mathrm{~nm}$ thin film produced by pulsed laser deposition from the sample, a nonstoichiometric mass transfer during PLD is evidenced. The calibration-free LIBS measurement procedure is expected to be applicable to thinner films if the film-composing elements are not present in the substrate.

\section{Acknowledgments}

Jacques Perrière is acknowledged for fruitful discussions on RBS analyses. The research leading to these results has received funding from LASERLAB-EUROPE (Grant Agreement No. 654148, European Union's Horizon 2020 research and innovation programme). EA and VC acknowledge the financial support from Romanian national projects NUCLEU-LAPLAS and STAR 161.

Supporting Information Available: Additional data and information on EDS and RBS analyses are given. This material is available free of charge via the Internet at http: //pubs.acs.org/.

\section{References}

(1) Dijkkamp, D.; Venkatesan, T.; Wu, X. D.; Shaheen, S. A.; Jisrawi, N.; Minlee, Y. H.; Mclean, W. L.; Croft, M. Appl. Phys. Lett. 1987, 51, 619-621.

(2) Sie, S. H. In Surface analysis methods in materials science; O'Connor, D. J., Sexton, B. A., Smart, R. S. C., Eds.; Springer, Berlin, 2003; Vol. 23; pp 229-246. 
(3) Jeynes, C.; Barradas, N. P.; Szilágyi, E. Anal. Chem. 2012, 84, 6061-6069.

(4) Kibel, M. H. In Surface analysis methods in materials science; O'Connor, D. J., Sexton, B. A., Smart, R. S. C., Eds.; Springer, Berlin, 2003; Vol. 23; pp 175202.

(5) Olesik, J. W. Anal. Chem. 1991, 63, A12A21.

(6) Groh, S.; Diwakar, P. K.; Garcia, C. C.; Murtazin, A.; Hahn, D. W.; Niemax, K. Anal. Chem. 2010, 82, 2568-2573.

(7) Cahoon, E. M.; Almirall, J. R. Anal. Chem. 2012, 84, 2239-2244.

(8) Climent-Font, A.; FernándezJiménez, M. T.; Wätjen, U.; Perrière, J. Nucl. Instr. Meth. Phys. Res. A 1994, 353, 575-578.

(9) Cabalin, L. M.; Laserna, J. J. Anal. Chem. 2001, 73, 1120-1125.

(10) In, J.-H.; Kim, C.-K.; Lee, S.-H.; Choi, J.H.; Jeong, S. Thin Solid Films 2015, 579, 89-94.

(11) Widjonarko, N. E.; Perkins, J. D.; Leisch, J. E.; Parilla, P. A.; Curtis, C. J.; Ginley, D. S.; Berry, J. J. Rev. Sci. Instrum. 2010, 81, 073103 1-8.

(12) Axente, E.; Hermann, J.; Socol, G.; Mercadier, L.; Beldjilali, S. A.; Cirisan, M.; Luculescu, C. R.; Ristoscu, C.; Mihailescu, I. N.; Craciun, V. J. Anal. At. Spectrom. 2014, 29, 553-564.

(13) Davari, S. A.; Hu, S.; Pamu, R.; Mukherjee, D. J. Anal. At. Spectrom. 2017, 32, 1378-1387.

(14) Ciucci, A.; Corsi, M.; Palleschi, V.; Rastelli, S.; Salvetti, A.; Tognoni, E. Appl. Spectrosc. 1999, 53, 960-964.

(15) Bulajic, D.; Corsi, M.; Cristoforetti, G.; Legnaioli, S.; Palleschi, V.; Salvetti, A.; Tognoni, E. Spectrochim. Acta Part B: Atom. Spectrosc. 2002, 57, 339-353.
(16) Hermann, J.; Grojo, D.; Axente, E.; Gerhard, C.; Burger, M.; Craciun, V. Phys. Rev. E 2017, 96, 053210 1-6.

(17) Mao, X. L.; Ciocan, A. C.; Russo, R. E. Appl. Spectrosc. 1998, 52, 913-918.

(18) Craciun, D.; Bourne, G.; Socol, G.; Stefan, N.; Dorcioman, G.; Lambers, E.; Craciun, V. Appl. Surf. Sci. 2011, 25\%, 5332-5336.

(19) Cooper, J. Rep. Prog. Phys. 1966, 29, 35130.

(20) Gerhard, C.; Hermann, J.; Mercadier, L.; Loewenthal, L.; Axente, E.; Luculescu, C. R.; Sarnet, T.; Sentis, M.; Viöl, W. Spectrochim. Acta Part B: Atom. Spectrosc. 2014, 101, 32-45.

(21) Cirisan, M.; Cvejić, M.; Gavrilović, M. R.; Jovicević, S.; Konjević, N.; Hermann, J. J. Quant. Spectrosc. Radiat. Transfer 2014, 133, 652-662.

(22) Burger, M.; Hermann, J. Spectrochim. Acta Part B: Atom. Spectrosc. 2016, 122, 118-126.

(23) Kramida, A.; Ralchenko, Y.; Reader, J. NIST Atomic Spectra Database (version 5.5.6). 2018; http://physics.nist.gov/ asd, National Institute of Standards and Technology, Gaithersburg, MD.

(24) Smith, P. L.; Heise, C.; Esmond, J. R.; Kurucz, R. L. Atomic spectral line database built from atomic data files from R. L. Kurucz CD-ROM 23. 2011; http://www.pmp.uni-hannover.de/ cgi-bin/ssi/test/kurucz/sekur.html.

(25) Hermann, J.; Lorusso, A.; Perrone, A.; Strafella, F.; Dutouquet, C.; Torralba, B. Phys. Rev. E 2015, 92, 053103 1-15.

(26) Cristoforetti, G.; Lorenzetti, G.; Legnaioli, S.; Palleschi, V. Spectrochim. Acta Part B: Atom. Spectrosc. 2010, 65, 787796. 
(27) Hermann, J.; Axente, E.; Craciun, V.; Taleb, A.; Pelascini, F. Spectrochim. Acta Part B: Atom. Spectrosc. 2018, 143, 6370.

(28) Tognoni, E.; Palleschi, V.; Corsi, M.; Cristoforetti, G.; Omenetto, N.; Gornushkin, I.; Smith, B. W.; Winefordner, J. D. In Laser-induced breakdown spectroscopy; Miziolek, A. W., Palleschi, V., Schechter, I., Eds.; Cambridge University, Berlin, 2006; pp 122194.

(29) Gonzalo, J.; Afonso, C. N.; Perrière, J. J. Appl. Phys. 1996, 79, 8042-8046.

(30) Ohnishi, T.; Lippmaa, M.; Yamamoto, T.; Meguro, S.; Koinuma, H. Appl. Phys. Lett. 2005, 87, 241919 1-3.

(31) Klamt, C.; Dittrich, A.; Jaquet, B.; Eberl, C.; Doering, F.; Krebs, H.-U. Appl. Phys. A: Mat. Sci. Proc. 2016, 122.

(32) Itina, T. E. J. Appl. Phys. 2001, 89, 740746.

(33) Droubay, T. C.; Qiao, L.; Kaspar, T. C.; Engelhard, M. H.; Shutthanandan, V.; Chambers, S. A. Appl. Phys. Lett. 2010, 97. 